\title{
Atomic resolution mapping of inequivalent $O$ sites in complex oxides
}

M. Varela*, A. R. Lupini*, M. P. Oxley***, W. Luo***, M. Watanabe***, J. T. Luck*, M. D. Biegalski****, H. M. Christen****, M. Murfitt*****, N. Dellby*****, O. Krivanek*****, S. T. Pantelides**,*, S. J. Pennycook****

*Oak Ridge National Laboratory, Materials Science \& Technology Division. Oak Ridge, TN, U.S.A.

**Vanderbilt University. Nashville, TN, U.S.A.

***Lehigh University. Bethlehem, PA, U.S.A.

****Oak Ridge National Laboratory, Center for Nanophase Materials Science. Oak

Ridge, TN, U.S.A.

*****Nion Co. Kirkland, Seattle. WA, U.S.A.

The reduction of electron probe sizes ensuing from aberration correction in the scanning transmission electron microscope (STEM) allows routine acquisition of atomic resolution electron energy loss spectroscopy (EELS) spectrum images. This makes STEM-EELS one of the most powerful analysis techniques of materials in real space, both at the nanoscale and at the atomic scale. A clear atomic lattice contrast can be now seen in elemental maps obtained from such images [1,2]. Figure 1 shows an example of a $\mathrm{CaTiO}_{3} / \mathrm{SrTiO}_{3}(\mathrm{CTO} / \mathrm{STO})$ interface, obtained in an aberration corrected Nion UltraSTEM column, operated at $100 \mathrm{kV}$. These images have been produced by conventional background subtraction using power law fits and integration of the remaining intensity under the edge [3]. The crystal lattices of $\mathrm{Ca}, \mathrm{Ti}$ and $\mathrm{O}$ are clearly resolved, and the different atomic columns can be identified unambiguously. A dislocation core can be observed at the interface. The element-sensitive imaging appears to show a decrease in $\mathrm{Ca}$ concentration and a local $\mathrm{Ti}$ enrichment at the dislocation core, which is non-stoichiometric $[3,4,5]$.

Here, we will apply atomic resolution EELS spectrum imaging combined with density functional theory and dynamical simulations to these and other complex oxide materials and nanosystems, such as interfaces $[6,7]$. Possible artifacts in the images, due e.g. to specimen preparation, will be examined. Other interesting effects such as those of nonlocality or dechanneling giving rise to volcano-like contrast such as the one observed on the $\mathrm{O} \mathrm{K}$ image of $\mathrm{CaMnO}_{3}$ shown in Figure 2 will also be discussed, together with the possibility of distinguishing inequivalent $\mathrm{O}$ species in Jahn-Teller distorted perovskites such as $\mathrm{LaMnO}_{3}$. All of these effects must be taken into account when trying to interpret atomic resolution EELS images obtained with aberration corrected electron probes.

[1] M. Bosman et al., Phys. Rev. Lett. 99 (2007) 086102.

[2] K. Kimoto et al., Nature 450 (2007) 702.

[3] M.Varela et al., Int. J. Mater. Res. Submitted (2009)

[4] M. Kim et al., Phys. Rev. Lett. 86 (2001) 4056.

[5] R.F. Klie et al., Nature 435 (2005) 475.

[6] N. D. Browning et al., Nature 366 (1993) 143.

[7] M. Varela et al., Sol. State Elec. 47 (2003) 2245.

[8] M. Varela et al., Phys. Rev. B 79 (2009) 085117. 
[9] Research at ORNL was sponsored by the Division of Materials Sciences and Engineering and the Scientific User Facilities Division (MDB, HMC) of the US Department of Energy.
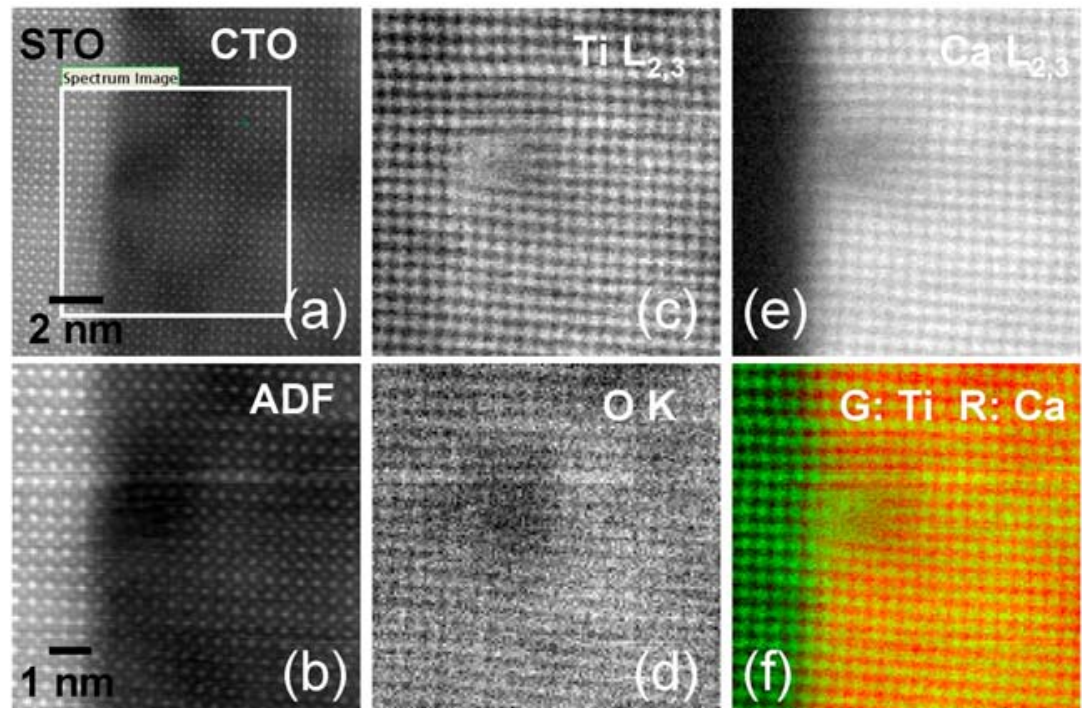

Figure 1: Spectrum imaging using the Nion UltraSTEM at $100 \mathrm{kV}$. (a) ADF image of a $\mathrm{CaTiO}_{3}$ (right) $/ \mathrm{SrTiO}_{3}$ (left) interface. A square marks the area where a 128 pixel $\mathrm{x}$ 128 pixel spectrum image was acquired, containing an interface dislocation core. (b) ADF signal acquired simultaneously with the SI. The nominal acquisition time was 0.01 s per pixel. (c) $\mathrm{Ti} \mathrm{L}_{2,3}$ map. (d) $\mathrm{O} \mathrm{K}$ map. (e) $\mathrm{Ca}_{2,3}$ map. These three elemental maps were produced by subtracting the background using a power law fit and integrating a 15 eV wide energy window below the edge. Principal Component analysis was applied to the EELS data to remove random noise. (f) Colored map produced by overlaying the Ti image (green) and the Ca image (red). Adapted from Ref. [3].

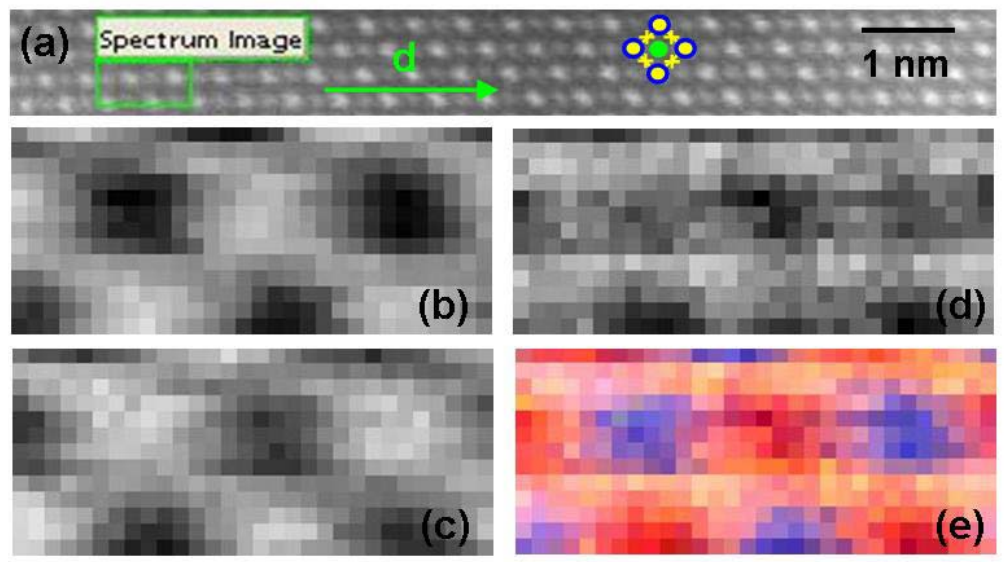

Figure 2. (a) Z-contrast image of a $\mathrm{CaMnO}_{3}$ perovskite down the pseudocubic axis obtained in an aberration corrected VG Microscopes HB501UX operated at $100 \mathrm{kV}$. The pseudocubic unit cell is marked (b) Ca L map, (c) Mn L map, and (d) O K map. (e) Color map produced from the overlay of the $\mathrm{Ca}$ (red), Mn (blue) and $\mathrm{O}$ (yellow) maps. Adapted from Ref [8]. 